\title{
High Intensity Focused Ultrasound Treatment-Induced Tumor Lysis Syndrome in Uterine Myoma Patient
}

\author{
Joon-Hyung Park', In-Mo Yang ${ }^{1}$, Young-Hwa Kim', Soo Jeong Yu', Jae Seong Lee², Do Hyoung Kim' \\ Departments of 'Internal Medicine and ${ }^{2}$ Obstetrics and Gynecology, Incheon Christian Hospital, Incheon, Korea
}

\begin{abstract}
Tumor lysis syndrome is one of the major oncological emergency. It causes complications such as acute kidney injury, cardiac arrhythmia, and death. However, it is rare in solid tumors. We report on a case of a patient with myoma of uterus who developed tumor lysis syndrome and acute kidney injury after high intensity focused ultrasound (HIFU) treatment. The potential complications of tumor lysis syndrome should be considered in HIFU treatment.
\end{abstract}

Keywords: Tumor lysis syndrome; High-intensity focused ultrasound; Myoma

\section{INTRODUCTION}

Tumor lysis syndrome (TLS) is characterized by hyperuricemia, hyperkalemia, hyperphosphatemia, and hypocalcemia resulting from the destruction of a large number of rapidly proliferating neoplastic cells in malignancy [1]. Cellular death mediated by cancer therapy (chemotherapy, other pharmacological antitumor intervention, or radiation therapy) or spontaneous cellular death in rapidly dividing tumors can lead to efflux of cellular material that is rich in potassium, phosphorus, and uric acid. Clinical complications such as seizures, acute kidney injury, cardiac arrhythmias, and death can occur in TLS patients. TLS is especially common in patients with hematological malignancies with rapid cellular turnover rates such as acute lymphocytic leukemia and Burkitt's lymphoma. However, TLS is very rare in patients with solid tumors [2]. In the present report, we describe a case of TLS occurred after high intensity focused ultrasound (HIFU) treatment in patient with uterine myoma.

\section{CASE REPORT}

A 35-year-old woman presented to Incheon Christian Hospital with back and abdominal pain occurred a few hours ago. She was treated with HIFU for myoma $(14 \times 10 \mathrm{~cm}$ in diameter) of the uterus in the past 5 days (Fig. 1). Her renal function was normal at that time. After HIFU treatment, mild abdominal discomfort was presented but controlled with non steroidal anti-inflammatory drug. Otherwise, her medical history was unremarkable.

At the time of presentation to our department, she had lower back pain without neurologic sign, lower abdominal pain without diarrhea, melena, or hematochezia. On physical examination, her blood pressure was $130 / 70 \mathrm{~mm} \mathrm{Hg}$ with heart rate of 70/min and body temperature of $36.5^{\circ} \mathrm{C}$. Lung sound was clear. Pitting edema was not detected. In laboratory test, her blood urea nitrogen level was $77 \mathrm{mg} / \mathrm{dL}$, creatinine level was $4.6 \mathrm{mg} / \mathrm{dL}$, estimated glomerular filtration rate was $11.7 \mathrm{~mL} / \mathrm{min} / 1.73 \mathrm{~m}^{2}$, serum sodium level was $135 \mathrm{mEq} / \mathrm{L}$, potassium level was $3.9 \mathrm{mEq} / \mathrm{L}$, calcium level was $7.6 \mathrm{mg} / \mathrm{dL}$, phosphorus level was $5.0 \mathrm{mg} / \mathrm{dL}$, uric acid level was
Correspondence to: Do Hyoung Kim

Department of Internal Medicine, Incheon Christian Hospital, 10 Dapdong-ro 30beon-gil, Jung-gu, Incheon 22320, Korea Tel: +82-32-270-8524, Fax: +82-32-270-8080, E-mail: dhkim6489@hanmail.net

Correspondence to: Jae Seong Lee

Department of Obstetrics and Gynecology, Incheon Christian Hospital, 10 Dapdong-ro 30beon-gil, Jung-gu, Incheon 22320, Korea

Tel: +82-32-270-8888, Fax: +82-32-270-8080, E-mail: flareljs@naver.com

Received: May 19, 2015 / Accepted after revision: Jul. 16, 2015
(C) 2015 Soonchunhyang Medical Research Institute This is an Open Access article distributed under the terms of the Creative Commons Attribution Non-Commercial License (http://creativecommons.org/licenses/by-nc/3.0/) 

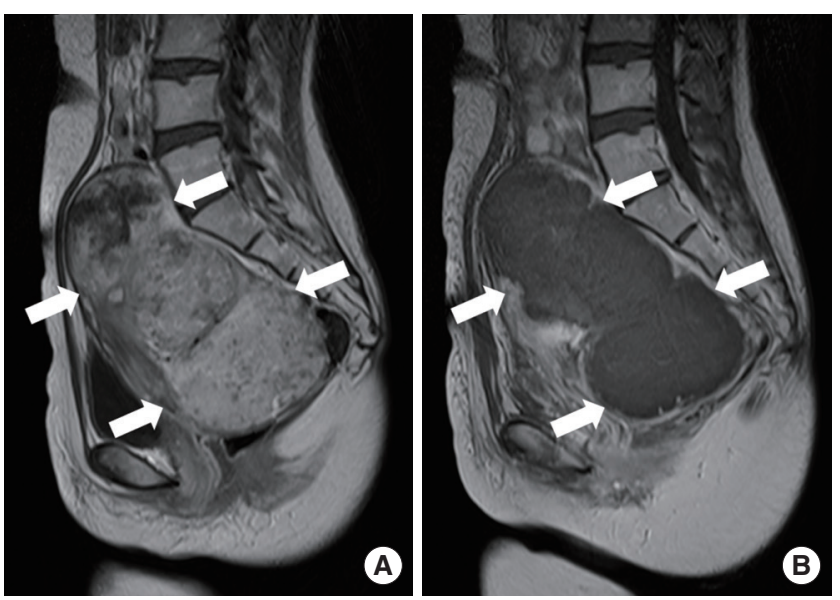

Fig. 1. Sagittal T1 weighted magnetic resonance images of uterine myoma (arrows) in the patient $(A)$ before and $(B)$ after high intensity focused ultrasound treatment showing signal change due to necrosis. (B) Note the decreased signal intensity in the entire lesion.

$11.3 \mathrm{mg} / \mathrm{dL}$, lactate dehydrogenase level was $709 \mathrm{IU} / \mathrm{L}$, and creatine kinase level was $274 \mathrm{U} / \mathrm{L}$. She had normal anion gap metabolic acidosis compensated by respiratory alkalosis. In urinalysis, she had trace amount of proteinuria. Her white blood cell (WBC) and red blood cell was 1-4/high-power field (HPF) and 5-9/HPF, respectively, with negative urine myoglobin. There was no evidence of hydronephrosis in kidney ultrasound. We considered that her symptoms had been associated with the inflammation in myoma caused by necrosis after HIFU treatment. However, in laboratory finding, acute kidney injury developed without symptoms to her. Considering that the patient had hyperuricemia, hyperphosphatemia, and acute kidney injury after HIFU treatment, TLS associated with HIFU treatment was suspected. She was treated by intravenous hydration at first. Her urine volume was maintained over $1 \mathrm{~L}$. She was administered furosemide and sodium bicarbonate by intravenous injection. She was also administered allopurinol. Because no symptom or sign that required hemodialysis was observed, hydration and treatment for TLS were maintained. In the next few days, the patient's clinical symptoms and signs such as lower abdominal, showed improvement, and biochemical markers including mild metabolic acidosis, hyperuricemia, hyperkalemia, hyperphosphatemia, and hypocalcemia returned to normal range (Fig. 2). The plasma level of lactate dehydrogenase was within near normal range. After 14 days of management and following up laboratory studies, her blood urea nitrogen concentration was decreased to $7 \mathrm{mg} / \mathrm{dL}$, creatinine concentration to $1.0 \mathrm{mg} / \mathrm{dL}$, lactate dehydrogenase to $4.9 \mathrm{mg} / \mathrm{dL}$, and

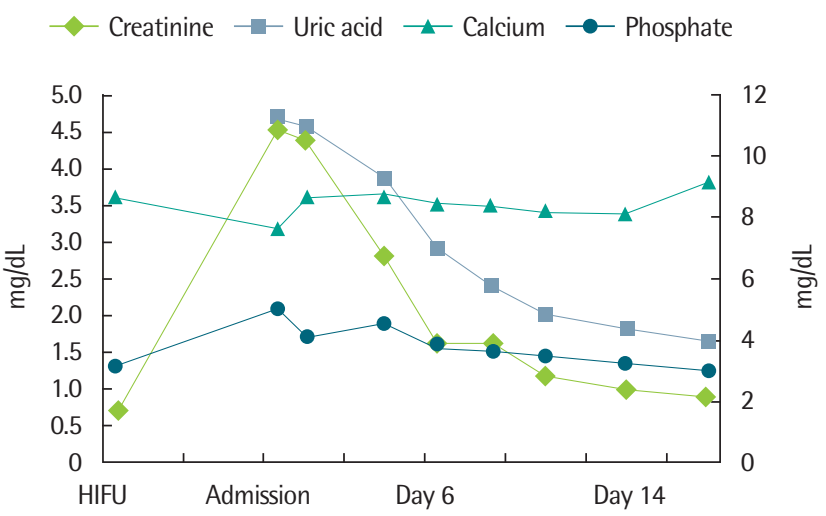

Fig. 2. Laboratory findings during hospitalization. HIFU, high intensity focused ultrasound.

uric acid concentration to $4.9 \mathrm{mg} / \mathrm{dL}$. The concentration of blood calcium and phosphorus was $8.1 \mathrm{mg} / \mathrm{dL}$ and $3.2 \mathrm{mg} / \mathrm{dL}$, respectively. She showed improvement in lower abdominal pain. The patient was discharged after 17 days of admission.

\section{DISCUSSION}

Uterine myomas are the most common benign neoplasm found in women of childbearing age. Uterine sparing treatment such as laparoscopic myomectomy and uterine artery embolization are common treatments for uterine myomas. However, they are associated with general anesthesia, hospitalization period, and lengthy recovery time. Recently, magnetic resonance imaging-guided focused HIFU as a non-invasive surgical procedure has been used worldwide to treat symptomatic uterine myoma over the past few years. Comparing the differences in intensities of HIFU and diagnostic ultrasound, HIFU has significantly higher time-averaged intensities (in the range of $100-10,000 \mathrm{~W} / \mathrm{cm}^{2}$ ) than typical diagnostic ultrasound (approximately $0.1-100 \mathrm{~mW} / \mathrm{cm}^{2}$ ) in the focal region of the ultrasound transducer [3]. However, the intensities are much lower between the transducer and the target so that the thermal damages are minimized outside the focal lesion. In the targeted tissue, the increased intensities will generate heat, raising temperature $\left(60^{\circ} \mathrm{C}\right.$ or higher) and causing coagulation necrosis [3]. In this case, extensive tissue necrosis was caused by HIFU treatment in huge uterine myoma. This necrosis induced TLS.

TLS is an oncologic emergency caused by massive tumor cell lysis with the release of large amounts of potassium, phosphate, and nucleic acids that are metabolized into uric acid [4]. TLS is commonly observed in hematological malignancies, such as acute lym- 
phocytic leukemia and Burkitt's lymphoma. However, TLS is uncommon in solid tumor [2,5]. The most important risk factor for TLS is malignancy, tumor burden (bulky lymphatic disease [ $>10$ $\mathrm{cm}$ ], elevated lactate dehydrogenase, and elevated WBC count). Other important risk factors include dehydration, baseline uric acid level, and preexisting renal insufficiency [6]. In our patient, there was no risk factor before HIFU treatment except tumor size. However, TLS was developed in benign neoplasm such as uterine myoma during HIFU treatment. To the best of our knowledge, HIFU treatment induced TLS in uterine myoma patient has not been previously reported.

Although no classification scheme for TLS is uniformly accepted, that of Cairo and Bishop is often used [7]. In the present case, the degree of abnormalities in her uric acid and phosphate fulfilled the criteria of TLS as defined by Cairo-Bishop. The criteria of clinical TLS were also satisfied by her creatinine level after HIFU treatment. The clinical and laboratory complications of TLS might be due to release of intracellular contents such as potassium, phosphorus, and nucleic acids into the extracellular space caused by tumor cell lysis after autonecrosis or chemotherapy. Released nucleic acids during cell lysis can be metabolized into hypoxanthine, then xanthine, and finally uric acid. Hyperkalemia can cause serious arrhythmias. Hyperphosphatemia can cause secondary hypocalcemia, leading to neuromuscular irritability, arrhythmia, and seizure. It can also precipitate as calcium phosphate crystals in various organs, including kidneys where these crystals can cause acute kidney injury. Uric acid can induce acute kidney injury not only by intrarenal crystallization but also by crystal-independent mechanisms such as renal vaso-constriction, impaired autoregulation, decreased renal blood flow, oxidation, and inflammation [8].

In conclusion, this was a case of TLS caused by HIFU treatment in uterine myoma which resulted in acute kidney injury. The risk factor of TLS in this patient was the huge size of myoma. Therefore, development of TLS should be considered when using HIFU to treat patient with large sized myoma.

\section{REFERENCES}

1. Wilson FP, Berns JS. Onco-nephrology: tumor lysis syndrome. Clin J Am Soc Nephrol 2012;7:1730-9.

2. Mirrakhimov AE, Ali AM, Khan M, Barbaryan A. Tumor lysis syndrome in solid tumors: an up to date review of the literature. Rare Tumors 2014; 6:5389.

3. Dubinsky TJ, Cuevas C, Dighe MK, Kolokythas O, Hwang JH. High-intensity focused ultrasound: current potential and oncologic applications. AJR Am J Roentgenol 2008;190:191-9.

4. Arrambide K, Toto RD. Tumor lysis syndrome. Semin Nephrol 1993;13: 273-80.

5. Howard SC, Jones DP, Pui CH. The tumor lysis syndrome. N Engl J Med 2011;364:1844-54.

6. Coiffier B, Altman A, Pui CH, Younes A, Cairo MS. Guidelines for the management of pediatric and adult tumor lysis syndrome: an evidencebased review. J Clin Oncol 2008;26:2767-78.

7. Cairo MS, Bishop M. Tumour lysis syndrome: new therapeutic strategies and classification. Br J Haematol 2004;127:3-11.

8. Shimada M, Johnson RJ, May WS Jr, Lingegowda V, Sood P, Nakagawa T, et al. A novel role for uric acid in acute kidney injury associated with tumour lysis syndrome. Nephrol Dial Transplant 2009;24:2960-4. 